\title{
Vergleichende Untersuchungen zur subpartalen Anwendung von Oxytocinpräparaten bei Sauen
}

\author{
Herrn Prof. Dr. habil. Peter Glodek zum 70. Geburtstag gewidmet
}

\begin{abstract}
Title of the paper: Comparative investigations into the subpartal application of Oxytocin preparations in sows

A veterinary supervision and monitoring of the farrowing process was carried out on a weaner production unit comprising of 1,500 sow places with a weekly ( 7 day) batch farrowing system. Farrowing was recorded as being interrupted in $15.2 \%$ of 1,975 farrowings as a result of inadequate contractions during labour and where the time-interval between the expulsion of consecutive piglets exceeded 40 minutes. 143 affected farrowing sows were allocated into three treatment groups and received an intramuscular injection of 20 I.U. Oxytocin (Group A, $\mathrm{n}=44$ ); $140 \mu \mathrm{g}$ of the agent Carbetocin (contained in $2 \mathrm{ml}$ of the long acting oxytocin preparation Depotocin ${ }^{\circledR}$; Group B, $n=47$ ) and respectively $70 \mu g$ Carbetocin per sow (Group C, $n=52$ ) to stimulate labour contractions. The oxytocin preparation was applied on average 148 minutes following the expulsion of the first piglet. On average 4.2 piglets had been born prior to this point in time. Following the injection of Carbetocin the time required for each piglet to be born, as well as the time to the completion of farrowing, were reduced significantly compared to Group A ( $<0.05$ ) and there was a tendency for a reduction in the proportion of piglets born dead. The various treatments had no fundamental influence on the proportion of those requiring manual assistance at farrowing. The labour contraction inducing injection of $70 \mu \mathrm{g}$ Carbetocin proved effective; this dosage rate is to be recommended for application during parturition in the pig.
\end{abstract}

Key Words: sow, birth, uterine inertia, oxytocin, carbetocin, expulsion of piglets

\section{Zusammenfassung}

In einem Ferkelerzeugerbetrieb mit 1.500 Sauenplätzen und Gruppenabferkelung im 7-Tage-Rhythmus erfolgte eine tierärztliche Kontrolle und Überwachung des Abferkelgeschehens. Bei 15,2 \% von 1.975 Schweinegeburten wurden Stockungen infolge von Wehenschwäche registriert, wodurch die Zeitspanne zwischen der Austreibung zweier aufeinanderfolgender Ferkel 40 Minuten überschritt. 143 betroffene Partussauen wurden auf drei Behandlungsgruppen aufgeteilt und erhielten als intramuskuläre Injektion 20 I.E. Oxytocin (Gruppe A, n = 44); 140 $\mu \mathrm{g}$ des Wirkstoffes Carbetocin (enthalten in $2 \mathrm{ml}$ des Langzeitoxytocin-Präparates Depotocin ${ }^{\circledR}$; Gruppe B, n = 47) resp. $70 \mu \mathrm{g}$ Carbetocin je Sau (Gruppe $C, \mathrm{n}=52$ ) zur Anregung der Wehentätigkeit. Die Applikation der Oxytocinpräparate erfolgte im Durchschnitt 148 Minuten nach der Expulsion des ersten Ferkels. Bis zu diesem Zeitpunkt waren im Mittel bereits 4,2 Ferkel je Wurf geboren. Nach der Injektion von Carbetocin wurden die Geburtsdauer je Ferkel sowie die Zeitspanne bis zum Geburtsende gegenüber Gruppe A signifikant $(p<0,05)$ und die Anteile tot geborener Ferkel tendenziell reduziert. Die unterschiedliche Behandlung hatte keinen wesentlichen Einfluss auf den Anteil der zu leistenden manuellen Geburtshilfe. Die wehenstimulierende Injektion von $70 \mu \mathrm{g}$ Carbetocin erwies sich als wirksam; die genannte Dosis ist für die Anwendung unter der Geburt beim Schwein empfehlenswert.

Schlüsselwörter: Sau, Geburt, Wehenschwäche, Oxytocin, Carbetocin, Expulsion der Ferkel

1. Einleitung

In der Sauenhaltung gehen immer mehr Betriebe zur Gruppenabferkelung über, um die Nachfrage nach größeren Ferkelpartien in marktgerechter Qualität zu befriedigen. Im 
Rahmen des Abferkelmanagements werden möglichst verdichtete Abferkelperioden der aufeinanderfolgenden Sauengruppen sowie eine kurze Geburtsdauer der Einzeltiere angestrebt (ZAREMBA und HÜHN, 1999; UDLUFT und BOSTEDT, 2003). Die hierauf ausgerichteten Maßnahmen schließen auch eine medikamentöse Beeinflussung des Geburtsgeschehens ein. In der veterinärmedizinischen Geburtshilfe und Gynäkologie ist Oxytocin ein häufig angewendetes Präparat.

Seit über einem Jahrzehnt steht neben Oxytocin dessen Langzeitformulierung Carbetocin - ein prolongiert wirkendes synthetisches Oxytocin-Analogon zur Beeinflussung der Geburt bei Rind und Schwein - zur Verfügung (EULENBERGER et al., 1993; KUDLAČ, 1993). Dieses Langzeitoxytocin gelangt vornehmlich im Rahmen der partiellen Partussynchronisation nach einer vorausgegangenen biotechnischen Behandlung mit Prostaglandin $\mathrm{F}_{2 \alpha}\left(\mathrm{PGF}_{2 \alpha}\right)$ zum Einsatz, um die noch ausstehenden Geburtseintritte der Sauen zum physiologischen Termin einzuleiten. Dadurch lässt sich eine unerwünschte Verlängerung der Tragezeit verhindern. Diese Methode trägt zur Verbesserung von tiergesundheitlichen Merkmalen der Partussauen und Ferkel sowie zur Aufzucht von ausgeglichenen Schweinewürfen bei (HÜHN und GEY, 1999; WÄHNER und HÜHN, 2001). Die Konzentration der Abferkeltermine einer Sauengruppe begünstigt die intensive Überwachung der Geburten sowie die Betreuung der Neugeborenen und erleichtert den Wurfausgleich zwischen Sauen mit unterschiedlicher Ferkelanzahl.

Sub partu bilden das Ausbleiben der Uteruskontraktionen und der Bauchpresse oder eine mangelhafte Wehentätigkeit häufige Ursachen für einen pathologischen Geburtsverlauf. Die Folgen äußern sich nicht selten in einer Verschleppung der Geburten, plazentarer Infektion, Absterben der pränatalen Früchte mit Störungen des Puerperiums und steigenden Ferkelverlusten (WENDT, 2002). Bei der sekundären Wehenschwäche lässt nach anfänglich normal verlaufender Geburt die Wehentätigkeit nach, so dass die Austreibung der Ferkel behindert ist und schließlich ganz ausbleibt. Unter der Voraussetzung, dass anderweitige Geburtshindernisse sicher auszuschließen sind, zielt die Therapie auf eine Anregung der Wehentätigkeit mit Oxytocika.

In den Ferkelerzeugerbetrieben wird Oxytocin in Dosen von vorzugsweise 20 I.E. intramuskulär appliziert. In der Regel setzt danach die Geburt spontan ein, gegebenenfalls ist eine manuelle Extraktionshilfe angebracht. Wegen der kurzen Halbwertzeit von 3 - 5 Minuten kann die Wirkung von Oxytocin bei langsamer Resorption aus Fettgewebe und gleichzeitigem Abbau ausbleiben. Oxytocin darf nicht überdosiert werden, damit es nicht zu Uterusspasmen und Geburtsstillstand kommt (PLONAIT, 2001).

Das synthetische Oxytocinanalogon Carbetocin ist bei intramuskulärer Applikation ebenso schnell und zuverlässig wirksam wie bei intravenöser. Die Halbwertzeit beträgt etwa 90 Minuten, die Wirkungsdauer der therapeutischen Dosis mehrere Stunden (REICHEL, 1988).

Damit ist eine Verlängerung der Uteruswirkung zu erzielen. Die seit Beginn der 90er Jahre gegebenen Dosierungsempfehlungen von 1,5 - 3,0 ml Depotocin ${ }^{\circledR}$ je Sau (entspricht 105 - $210 \mu \mathrm{g}$ Carbetocin/Sau) bildeten jahrelang die Basis der vielerorts gebräuchlichen Anwendungspraxis (BERNHARD et al., 1993). Zugleich erfuhr die im angegebenen Bereich liegende Hochdosierung von $210 \mu \mathrm{g}$ Carbetocin aufgrund guter Erfahrungen von Praktikern mit der niedrigeren Dosierungsstufe von $140 \mu \mathrm{g}$ eine kritische Bewertung. Eine weitere Reduzierung auf $70 \mu \mathrm{g}$ erschien aussichtsreich. Es 
galt im Weiteren zu prüfen, wie sich bei Sauen mit Geburtsverzögerungen infolge von Wehenschwäche eine Injektion von unterschiedlich dosiertem Depotoxytocin im Vergleich zu Oxytocin auf verschiedene Parameter des Geburtsverlaufes auswirkt. Die folgende Untersuchung sollte die Prüfung der Dosierungen von 70 bzw. $140 \mu \mathrm{g}$ Carbetocin/Tier im Vergleich zu 20 IE Oxytocin/Tier einschließen.

\section{Material und Methoden}

\section{Untersuchungsbetrieb}

Die Untersuchungen erfolgten in einem Ferkelerzeugerbetrieb mit 1.500 Sauenplätzen in Thüringen. Dieser verfügt über ein Raumprogramm mit voneinander getrennten Stallabteilen für besamte, für tragende sowie für säugende Sauen. Die Abferkel- und Aufzuchtställe werden strikt nach dem "Alles-rein-alles-raus"-Prinzip bewirtschaftet. Das Produktionszyklogramm des Betriebes basiert auf dem 7-Tage-Rhythmus in Verbindung mit einer vierwöchigen Säugezeit. Der Anteil erster Würfe an den Geburten insgesamt liegt bei ca. 25 \%. Es gelangen wöchentlich 50 bis 55 hochtragende Sauen zur Abferkelung. Im Untersuchungszeitraum standen 288 Abferkelbuchten zur Verfügung.

Im Untersuchungsbetrieb werden die Tiere nach den Regeln der guten landwirtschaftlichen Praxis gehalten. Die Fütterung erfolgte auf der Grundlage der Versorgungsempfehlungen der Fütterungsberatung unter Verwendung industriell hergestellter Alleinfuttermittel. Die Sauengruppen wurden mittels des praxisüblichen Verfahrens der Ovulationssynchronisation auf die künstliche Besamung vorbereitet. Diese erfolgte terminorientiert unter Verwendung von zwei Besamungsportionen $\left(\mathrm{KB}_{1}\right.$ und $\left.\mathrm{KB}_{2}\right)$.

Die hochtragenden Sauen wurden am 108. Trächtigkeitstag aus dem Wartestall in die gereinigten und desinfizierten Abferkelställe umgestellt. Sie verblieben dort in Einzelbuchten mit Ferkelschutzkorb bis zum Absetztermin.

Die Abferkelbuchten gliederten sich in die Bereiche Sauenstand, Fress- und Liegeplatz der Ferkel und Ausweichraum für Ferkel. Sie waren so beschaffen, dass die Ferkel gut an das Gesäuge der Sau gelangen konnten. Es war eine gute Übersicht gegeben und die Handhabung von Sau und Ferkeln war ohne Behinderung möglich. Für die Ferkel war ein gesonderter, mit zusätzlicher Wärmequelle ausgestatteter, ca. 0,60 - 0,70 $\mathrm{m}^{2}$ großer Liegebereich vorhanden. Die Futtervorlage für die Sau im Trog (vor dem Kopf angeordnet) wurde zweimal täglich vorgenommen. Die Wasserversorgung der Muttertiere und Ferkel erfolgte über Selbsttränken.

Bei denjenigen Sauen, die bis zu einem vorgegebenen Termin noch keine Geburtseintritte aufwiesen, wurde frühestens am Morgen des 114. Trächtigkeitstages mittels eines Cloprostenol-haltigen $\mathrm{PGF}_{2 \alpha}$-Präparates die Geburt eingeleitet.

\section{Tiermaterial}

Bei den Sauen handelte es sich um Tiere aus der Rotationskreuzung unter Verwendung von drei Mutterrassen (Deutsche Landrasse, Edelschwein und Leicoma). Sie wurden zum Zwecke der Mastferkelerzeugung mit Sperma von Besamungsebern der Rasse Pietrain besamt. Im Untersuchungszeitraum, welcher sich über 42 Wochengruppen erstreckte, wurden insgesamt 1.975 Schweinegeburten registriert und auf das Auftreten von Geburtsstockungen hin kontrolliert. Alle Partussauen wurden zuvor bei der Einstallung in den Abferkelstall klinisch untersucht. Offensichtlich kranke Tiere wurden nicht in den Versuch aufgenommen. In der Abferkelsektion standen die Partussauen 
unter ständiger Überwachung. Es traten bei insgesamt 301 Sauen Geburtsverzögerungen auf. Ihr Anteil betrug somit 15,2 \%. Es wurden nachfolgend von 143 überwachten Abferkelungen mit registrierten Geburtsstockungen sowie mit notwendigen Geburtshilfen Untersuchungsergebnisse zum Geburtsgeschehen (Übersicht) gewonnen. Als Geburtsstockung galten Überschreitungen einer Austreibungszeit zwischen zwei nacheinander geborenen Ferkeln von 40 Minuten. Nach einer registrierten Geburtsstockung wurde die wehenstimulierende Injektion vorgenommen.

Übersicht

Geprüfte Parameter von 143 Partussauen mit Geburtsverzögerungen (Assessment parameters for 143 farrowing sows with delayed births)

\begin{tabular}{|l|l|}
\hline Parameter & Definition \\
\hline Geburtseintritt & Zeitpunkt der Expulsion des ersten Ferkels eines Wurfes \\
\hline Geburtsdauer & $\begin{array}{l}\text { Entsprechend der Erfassbarkeit unter Praxisbedingungen } \\
\text { als Zeitdauer von der Expulsion des ersten Ferkels bis zum } \\
\text { letzten geborenen Ferkel eines Wurfes ausgewiesen. Bei } \\
\text { der Geburtsdauer erfolgte eine Unterteilung in die Ab- } \\
\text { schnitte vom Geburtseintritt bis zur Durchführung der we- } \\
\text { henstimulierenden Injektion sowie von der Injektion bis } \\
\text { zur Geburt des letzten Ferkels des betreffenden Wurfes. }\end{array}$ \\
\hline Austreibungszeit & $\begin{array}{l}\text { Quotient aus Geburtsdauer und Anzahl insgesamt gebore- } \\
\text { ner Ferkel je Wurf }\end{array}$ \\
\hline Wurfleistungen & $\begin{array}{l}\text { Anzahl geborener Ferkel je Wurf, getrennt nach insgesamt, } \\
\text { lebend sowie tot geborenen Ferkeln (absolut und in \%, } \\
\text { letzteres bezogen auf insgesamt geborene Ferkel). } \\
\text { Bei den insgesamt geborenen Ferkeln erfolgte eine Zuord- } \\
\text { nung zu den Geburtsabschnitten vom Geburtseintritt bis } \\
\text { zur wehenstimulierenden Injektion sowie von der Injektion } \\
\text { bis zum Geburtsende }\end{array}$ \\
\hline Reaktionszeit auf die wehenstimulierende & $\begin{array}{l}\text { Zeitspanne von der wehenstimulierenden Injektion bis zum } \\
\text { Geburtsende }\end{array}$ \\
\hline Injektion & $\begin{array}{l}\text { Erfassung der Sauen, bei denen nach der wehenstimulie- } \\
\text { renden Injektion erneut eine Geburtsstockung eintrat, die } \\
\text { manuelle Extraktionshilfe erforderte }\end{array}$ \\
\hline
\end{tabular}

Es erfolgte eine Aufteilung der 143 Tiere mit Geburtsstockungen auf drei Behandlungsgruppen:

Sauengruppe A: $n=44$, i.m. (intramuskuläre) Applikation von 20 IE Oxytocin

Sauengruppe B: $\mathrm{n}=47$, i.m. Applikation von $140 \mu \mathrm{g}$ Carbetocin

Sauengruppe C: $\mathrm{n}=52$, i.m. Applikation von $70 \mu$ g Carbetocin

Zum Einsatz kam jeweils eine Charge von Oxytocin und Depotocin ${ }^{\circledR}$ (Veyx-Pharma GmbH, Schwarzenborn).

\section{Auswertung der Versuche}

Alle an den Einzeltieren erfassten Daten wurden dokumentiert (Sauenkarten). Die Versuchsplanung und die statistische Bearbeitung erfolgten unter Zugrundelegung eines Signifikanzniveaus von $\mathrm{p}<0,05$. Als statistische Lageparameter wurden der arithmetische Mittelwert $(\bar{x})$ und die Standardabweichung $( \pm s)$ berechnet. Zur Prü- 
fung der statistischen Signifikanz kam der Student-Test zur Anwendung. Die Verteilungsunterschiede von Ereignishäufigkeiten wurden mittels 2 x 2-Feldertafeln und dem $\mathrm{Chi}^{2}$-Test nach Pearson auf Signifikanz geprüft.

\section{3. $\quad$ Ergebnisse}

Die auf die drei Behandlungsgruppen A, B und C aufgeteilten Sauen erreichten die in Tabelle 1 ausgewiesenen durchschnittlichen Wurfleistungen. Die Mittelwerte unterschieden sich nur zufällig voneinander. In der Oxytocin-Gruppe waren die Anzahl sowie der Anteil tot geborener Ferkel am höchsten, jedoch ließen sich die Differenzen zu den Carbetocin-Gruppen beim vorliegenden Stichprobenumfang statistisch nicht sichern.

Tabelle 1

Sauengruppen mit medikamenteller Geburtshilfe und deren Wurfleistungen (Medicated obstetrics of sow groups and their litter performance)

\begin{tabular}{ccccccc}
\hline $\begin{array}{c}\text { Sauen- } \\
\text { gruppe }\end{array}$ & $\begin{array}{c}\text { wehenstimulierende } \\
\text { Injektion }\end{array}$ & Sauen & \multicolumn{2}{c}{ geborene Ferkel je Wurf } & \multicolumn{2}{c}{ tot geb. Ferkel } \\
ins & ingesamt & lebend & tot & \% \\
\hline A & 20 IE Oxytocin & 44 & 11,50 & 10,18 & 1,32 & 11,5 \\
B & $140 \mu$ g Carbetocin & 47 & 10,47 & 9,49 & 0,98 & 9,4 \\
C & $70 \mu$ g Carbetocin & 52 & 10,88 & 10,02 & 0,86 & 9,2 \\
\hline
\end{tabular}

Bei den in die vergleichenden Untersuchungen einbezogenen 143 Schweinegeburten wurde nach registrierter Geburtsstockung die wehenstimulierende Injektion im Mittel 148 Minuten nach der Austreibung des ersten Ferkels eines Wurfes vorgenommen. Bis zum Zeitpunkt dieser Injektion waren durchschnittlich bereits 4,2 Ferkel je Wurf geboren. Ihnen folgten im weiteren Geburtsverlauf 6,26 geborene Ferkel. Die Gruppen A, $\mathrm{B}$ und $\mathrm{C}$ variierten nur zufallsbedingt um diese Mittelwerte.

Tabelle 2

Geburtsdauer der Sauengruppen mit unterschiedlicher medikamenteller Geburtshilfe (Duration of birth in the sow groups for the various medicated obstetric treatments)

\begin{tabular}{|c|c|c|c|c|c|c|c|c|c|}
\hline \multirow{3}{*}{$\begin{array}{l}\text { Sauen- } \\
\text { gruppe }\end{array}$} & \multirow[t]{3}{*}{$\mathrm{n}$} & \multicolumn{4}{|c|}{ Geburtsdauer (min) } & \multicolumn{4}{|c|}{ Geburtsdauer je geb. Ferkel (min) } \\
\hline & & \multicolumn{2}{|c|}{ insgesamt } & \multicolumn{2}{|c|}{ je geb. Ferkel } & \multicolumn{2}{|c|}{ vor Geburtshilfe } & \multicolumn{2}{|c|}{ nach Injektion } \\
\hline & & $\bar{x}$ & $\mathrm{~S}$ & $\bar{x}$ & $\mathrm{~S}$ & $\bar{x}$ & $\mathrm{~s}$ & $\bar{x}$ & $\mathrm{~s}$ \\
\hline A & 44 & 302,7 & 87,4 & 26,3 & 11,7 & 42,9 & 31,8 & $31,9^{\mathrm{a}}$ & 23,6 \\
\hline B & 47 & 278,3 & 94,5 & 26,5 & 16,8 & $46,5^{\mathrm{A}}$ & 42,5 & $22,9^{\mathrm{B}}$ & 25,8 \\
\hline $\mathrm{C}$ & 52 & 269,2 & 84,0 & 24,7 & 8,9 & $41,2^{\mathrm{A}}$ & 24.6 & $20,4^{\mathrm{B}, \mathrm{b}}$ & 11,1 \\
\hline
\end{tabular}

a, b - ungleiche Buchstaben in der gleichen Spalte bezeichnen signifikante Differenzen $(\mathrm{p}<0,05)$

A, B - ungleiche Buchstaben in der gleichen Zeile bezeichnen signifikante Differenzen $(\mathrm{p}<0,05)$

Die Geburten dauerten bei den Sauen der Gruppe A länger als bei denen der Gruppen B und C (nicht signifikant). In allen Behandlungsgruppen wirkten die Injektionen beschleunigend auf die darauffolgende Austreibungszeit je geborenes Ferkel (Tab. 2). Bei den Carbetocin-Gruppen war diese Wirkung signifikant. Die niedriger dosierte Carbetocin-Gruppe C war der Oxytocin-Gruppe A bedeutend überlegen. Die nach der Injektion geborenen Ferkel wiesen die kürzeste mittlere Austreibungszeit auf, und bei ihnen streute die Geburtsdauer deutlich weniger als in den beiden anderen Gruppen.

Von der wehenstimulierenden Injektion bis zur Austreibung des letzten Ferkels, d. h. bis zum Geburtsende, verging in Gruppe A eine signifikant längere Zeitspanne als in 
den beiden Carbetocin-Gruppen. Die Schwankungsbreite zwischen den Probanden war deutlich größer als in den Gruppen B und C (Tab. 3).

Tabelle 3

Zeitspanne von der wehenstimulierenden Injektion bis Geburtsende sowie Anteil zusätzlicher manueller Geburtshilfen (Time span from the giving of the labour contraction stimulating injection through to the completion of parturition, as well as the additional proportion requiring manual farrowing assistance)

\begin{tabular}{cccccccc}
\hline $\begin{array}{c}\text { Sauen- } \\
\text { gruppe }\end{array}$ & \multirow{2}{*}{} & \multicolumn{3}{c}{ Abstand Injektion - Geburtsende (min) } & \multicolumn{2}{c}{ Geburten mit manueller Hilfe } \\
& & $\overline{\mathrm{x}}$ & $\mathrm{s}$ & Min. & Max. & Anzahl & $\%$ \\
\hline A & 44 & $176,2^{\mathrm{a}}$ & 91,0 & 40 & 480 & 20 & 45,5 \\
B & 47 & $99,0^{\mathrm{b}}$ & 67,0 & 0 & 330 & 15 & 31,9 \\
C & 52 & $122,7^{\mathrm{b}}$ & 72,8 & 10 & 350 & 22 & 42.3 \\
\hline
\end{tabular}

Bei 57 von 143 stockenden Geburten wurde nach vergeblicher medikamenteller Intervention manuelle Geburtshilfe geleistet. Das ergab einen Anteil von 39,9 \%. Die in Tabelle 3 ausgewiesenen Gruppenunterschiede variierten nur zufallsbedingt um diesen mittleren Anteil.

Schweinegeburten, deren Fortgang einer zusätzlichen manuellen Hilfe bedurfte (Extraktion eines oder mehrerer Ferkel), wiesen eine längere Geburtsdauer je Ferkel auf als solche, bei denen sich eine wehenstimulierende Injektion allein als ausreichend erwies (Tab. 4). Bei der zuletzt genannten Kategorie war die Carbetocin-Anwendung signifikant überlegen gegenüber Oxytocin. Bei der Kategorie "medikamentelle und manuelle Hilfe" bestand eine tendenzielle Vorzugsstellung der Niedrigdosierung von Carbetocin hinsichtlich der mittleren Austreibungszeit je geborenes Ferkel.

Tabelle 4

Geburtsdauer je Ferkel nach Durchführung geburtshilflicher Maßnahmen (Time duration for the birth of each piglet following the carrying out of the obstetric procedures)

\begin{tabular}{|c|c|c|c|c|c|c|c|}
\hline \multirow[t]{2}{*}{$\begin{array}{l}\text { Sauen- } \\
\text { gruppe }\end{array}$} & \multirow[t]{2}{*}{$\mathrm{n}$} & \multicolumn{3}{|c|}{$\begin{array}{l}\text { Geburtsdauer je Ferkel (min) } \\
\text { nach alleiniger Injektion }\end{array}$} & \multicolumn{3}{|c|}{$\begin{array}{l}\text { Geburtsdauer je Ferkel (min) nach } \\
\text { Injektion und manueller Hilfe }\end{array}$} \\
\hline & & Würfe & $\bar{x}$ & $\mathrm{~s}$ & Würfe & $\bar{x}$ & s \\
\hline A & 44 & 24 & $28,4^{\mathrm{a}}$ & 13,0 & 20 & 36,1 & 31,5 \\
\hline B & 47 & 32 & $16,9^{\mathrm{b}, \mathrm{A}}$ & 10,4 & 15 & $35,5^{\mathrm{B}}$ & 40,2 \\
\hline C & 52 & 30 & $17,8^{\mathrm{b}}$ & 9,7 & 22 & 23,9 & 11,9 \\
\hline
\end{tabular}

a, b - ungleiche Buchstaben in der gleichen Spalte bezeichnen signifikante Differenzen $(\mathrm{p}<0,05)$

A, B - ungleiche Buchstaben in der gleichen Zeile bezeichnen signifikante Differenzen $(\mathrm{p}<0,05)$

\section{4.}

\section{Diskussion}

In den Schweinezuchten und Ferkelerzeugerbetrieben soll das Abferkelmanagement sicherstellen, dass die gebärenden Tiere geschützt werden und ihnen bei Geburtsstörungen sachgerechte Hilfe zuteil wird. Eine Geburtenüberwachung ermöglicht es, Geburtsstörungen rechtzeitig zu erkennen, wodurch sich peripartale Verluste verringern lassen und die postnatale Umweltanpassung der Neugeborenen gefördert wird (PRANGE, 2001). Die Etablierung periodenweiser Abferkelsysteme und der gezielte Einsatz fortpflanzungssteuernder Maßnahmen schaffen für die intensive Überwachung und Betreuung der Partustiere vorteilhafte Bedingungen (KÖNIG und HÜHN, 1997). Einer Auswertung zufolge, welche anlässlich des im Frühjahr 2003 in Fulda durchgeführten Forums "Spitzenbetriebe Ferkelproduktion" der Deutschen Landwirtschaftsge- 
sellschaft zum Schwerpunkt Tiergesundheit vorgenommen wurde (REIMANN, 2003), wendeten 49 \% der befragten Ferkelerzeuger die Geburtensynchronisation an. $41 \%$ verzichteten darauf; der Rest machte keine Angaben. Der Anteil überwachter Geburten lag bei 58 \% der sauenhaltenden Betriebe. Im Untersuchungsbetrieb erfolgte die Gruppenabferkelung der Sauen im einwöchigen Rhythmus. Die Hauptabferkelperioden erstreckten sich auf drei Werktage. Es bestand eine durchgängige, tierärztlich begleitete Geburtsüberwachung durch geeignete Fachkräfte. Während des 42wöchigen Untersuchungszeitraumes wurden bei 15,2 \% von insgesamt 1.975 kontrollierten Schweinegeburten Stockungen registriert, die geburtshilfliche Maßnahmen erforderlich machten. Eine frühere Analyse hatte im gleichen Betrieb bei 28,1 \% von 576 mittels biotechnischer Maßnahmen gesteuerten Geburten auftretende Wehenschwäche ergeben. Bei 11,1 \% der Partustiere wurde manuelle Geburtshilfe geleistet (UDLUFT, 1988). Nach den von BUSCH (1993) vermittelten Erfahrungen mussten in ostdeutschen Großbetrieben z. B. 30 \% aller gebärenden Sauen wegen stockenden Fortgangs der Geburt bzw. zur Ermittlung des beendeten Geburtsvorganges vaginal untersucht werden und mindestens $20 \%$ der Tiere erhielten wehenfördernde Injektionen. HOY (2002) registrierte in einem Betrieb mit 28,5 \% puerperalerkrankten Sauen tendenziell weniger Geburtsstörungen bei den gesunden Tieren (11,6 \%) im Vergleich zu den Zeitgefährtinnen mit MMA(Mastitis-Metritis-Agalaktie)-Syndrom (17,1 \%).

PRANGE (2002) analysierte in einer großen Anzahl von Beständen die auftretenden Schwankungen des Geburtsgeschehens sowie die damit verbundenen Leistungsparameter und leitete daraus Norm- und Orientierungswerte ab. Demnach ist die mittlere Geburtsdauer je Wurf auf zwei bis vier Stunden (Schwankungsbreite 0,5 bis 8 Stunden) zu beziffern und von Geburtsstörungen sind im Durchschnitt unter $5 \%$ der Jungsauen sowie unter $10 \%$ der Altsauen betroffen.

Nach SCHULZ (2002) werden die natürlichen Variationsbreiten für die Geburtsdauer mit 25 Minuten bis 8 Stunden, für die Abstände zwischen zwei aufeinanderfolgenden Ferkelgeburten mit einer Minute bis zu mehreren Stunden angegeben. Im Untersuchungsbetrieb wurden übereinstimmend mit früheren Studien (SPITSCHAK und HÜHN, 1994; ZAREMBA und HÜHN, 1999) alle spontanen Geburten bis zum 114. Trächtigkeitstag abgewartet und bei den Sauen mit noch ausstehender Abferkelung frühestens ab diesem Termin eine partielle Geburtsauslösung mittels eines Cloprostenol-haltigen Prostaglandin $\mathrm{F}_{2 \alpha}$-Präparates vorgenommen. Diejenigen Sauen, die nicht innerhalb von 24 Stunden nach der Cloprostenol-Injektion Geburtseintritte aufwiesen, erhielten zur Vermeidung einer Übertragung sowie zur terminlichen Konzentration der Abferkelungen eine Injektion des Wirkstoffes Carbetocin (enthalten im Depotoxytocin). Die Art der Geburtsauslösung hatte nach einem Bericht von UDLUFT und BOSTEDT (2004) einen signifikanten Einfluss auf die mittlere Geburtsdauer und deren Streuung: Diese betrug bei 585 spontan abferkelnden Sauen $240 \pm$ 83,7 Minuten; bei 526 Sauen nach Cloprostenol-Injektion $230 \pm 82,4$ Minuten. Die Sauen mit kombinierter Cloprostenol + Carbetocin $(70 \mu \mathrm{g} / \mathrm{Tier})$-Behandlung $(\mathrm{n}=155)$ hatten eine deutlich kürzere Geburtsdauer (186 \pm 57,5 Minuten) und wiesen einen ebenfalls signifikant $(\mathrm{p}<0,05)$ reduzierten Anteil tot geborener Ferkel (5,3 \%) gegenüber den Tieren mit Spontangeburten (8,9\%) bzw. nach Cloprostenol-Injektion (6,6 \%) auf.

Die Wirkung des Carbetocins übertraf diejenige von Oxytocin (20 I.E. je Sau), welches in einer Vergleichsgruppe $(n=134)$ eine Geburtsdauer von $209 \pm 68,2$ Minuten sowie 7,1 \% tot geborene Ferkel zur Folge hatte (UDLUFT und BOSTEDT, 2004). 
Ein zügiger Geburtsverlauf ist für die Gesundheit von Sauen und Ferkeln gleichermaßen bedeutsam. Daher erwies es sich als zweckmäßig, Sauen mit Wehenschwäche medikamentell zu behandeln. Zur generellen Beschleunigung der Schweinegeburt wurde in früherer Zeit am häufigsten Oxytocin eingesetzt (ELLENDORF, 1980; LEHMANN, 1984). In konzentrierten Sauenbeständen hat sich vielfach die Injektion von Oxytocin im letzten Geburtsdrittel bewährt (DANNENBERG, 1986). Bei der vorliegenden Studie, welche 143 Partussauen mit Geburtsstockungen umfasste, erfolgte der Einsatz von Oxytocin bzw. Depotoxytocin erst dann, wenn sich im Verlauf der überwachten Schweinegeburten die Austreibungszeit zwischen zwei Ferkeln auf über 40 Minuten verlängerte.

PRANGE (2002) empfahl, eine metaphylaktische Oxytocinapplikation in Problembeständen generell bzw. bei Problemtieren besser erst nach begonnener Geburt als ante partum durchzuführen. Als günstig wurde der Abschnitt zwischen dem 4. und 7. geborenen Ferkel herausgestellt. Nach dänischen Erfahrungen kann ein häufiger Gebrauch von Oxytocin einen Krampf oder eine Erschöpfung der Sau nach sich ziehen und auch vermehrt Totgeburten verursachen (THORUP, 2002). Hiervon abweichend, gelangte nach einem Praxisbericht in zahlreichen industriell geführten Schweinezuchtbeständen Südosteuropas mit bewegungsarm gehaltenen Muttersauen Oxytocin routinemäßig nach der Geburt des ersten Ferkels zum Einsatz, und die tierärztlich betreuten Großbestände erreichten hierdurch eine Senkung der frühpostnatalen Ferkelverluste, weniger an MMA erkrankte Sauen und höhere 4-Wochen-Gewichte der Ferkel (BILKEI, 1993). In neueren wissenschaftlichen Untersuchungen hatten die Sauen, denen in Abhängigkeit von ihrer Lebendmasse bei Geburtsbeginn 20, 40 oder 60 I.E. Oxytocin injiziert worden waren, nachfolgend eine erhöhte Rate an fetalem Distress, Mangel an Sauerstoff in den Geweben der Neugeborenen und vermehrte intrapartale Verluste (MOTAROJAS et al., 2002).

Die subpartale Verabreichung von Carbetocin anstelle von Oxytocin versprach wegen der verlängerten und damit intensiveren Wirkung des Langzeitoxytocins (LEHMANN, 1984; EULENBERGER et al., 1993) einen vorteilhaften Einfluss auf die nachfolgende Geburtsdauer. Die diesbezüglich vergleichend geprüften Dosierungen orientierten sich an Literaturmitteilungen. In der Wirkung an der gesunden Sau soll 1,0 mg Carbetocin 50 I.E. Oxytocin entsprechen (PLONAIT, 2001). In vorausgegangenen experimentellen Studien zur gleichen Fragestellung wurde die biologische Aktivität von 0,07 mg Carbetocin als äquivalent zu 10 I.E. Oxytocin bezeichnet. Als optimaler Dosisbereich wurden zur Beschleunigung der Geburtsdauer 0,14 bis 0,21 mg Carbetocin je Sau anstelle von 20 I.E. Oxytocin herausgestellt (MAFFEO et al., 1990). Die Ergebnisse neuerer Untersuchungen zur möglichen Dosisreduzierung von Depotoxytocin im Rahmen kombinierter Behandlungsprogramme zur partiellen Geburtensynchronisation legten es nahe, den Einsatz von $140 \mu \mathrm{g}$ sowie von $70 \mu \mathrm{g}$ Carbetocin vergleichend $\mathrm{zu}$ prüfen (HÜHN und GEY, 1999; UDLUFT und BOSTEDT, 2003).

In der Tat führte die Injektion von Carbetocin bei Sauen mit Geburtsstockungen zur Wehenstimulation, die Geburtsdauer wurde verringert und ein früheres Geburtsende erreicht. Diese Wirkungen waren zwischen beiden Carbetocindosierungen nahezu gleich und übertrafen diejenigen nach Injektion des herkömmlichen Oxytocins (20 I.E. je Partussau) deutlich $(\mathrm{p}<0,05)$. Bei einem Teil der Sauen reichte die geburtsbeschleunigende Wirkung nach der alleinigen Applikation eines Oxytocinpräparates nicht aus, und es musste zusätzlich manuelle Hilfe geleistet werden. Dies betraf im 
Durchschnitt 39,9 \% der Partussauen mit vorangegangener Geburtsstockung. THORUP (2002) bezifferte für die dänischen Ferkelerzeugerbetriebe den Anteil an Sauen, die normalerweise eine menschliche Geburtshilfe erfahren, auf 20 \%. Im vorliegenden Falle erreichten die Sauen nach Injektion von $70 \mu \mathrm{g}$ Carbetocin und manueller Geburtshilfe eine kürzere Geburtsdauer je Ferkel als die beiden anderen Varianten.

Die Ergebnisse der Untersuchungen lassen folgende Schlussfolgerungen zu:

1. Unter den vorliegenden Bedingungen erwies sich die wehenstimulierende Injektion von $70 \mu \mathrm{g}$ Carbetocin als wirksam. Bei Partussauen mit Geburtsstockungen wurde der Fortgang der Geburten angeregt.

2. Die genannte Dosierung war der bislang vornehmlich praktizierten Variante "Injektion von $140 \mu \mathrm{g}$ Carbetocin" gleichwertig und gilt als empfehlenswert. Hierdurch wird der Wirkstoffeinsatz je Partussau in wünschenswerter Weise reduziert.

3. Die Applikation von Carbetocin erbrachte günstigere Ergebnisse für den Geburtsverlauf und die Totgeburtenrate der Ferkel als der Einsatz von Oxytocin. Neben dem biotechnischen Einsatz von Prostaglandinen steht mit dem Langzeitoxytocin ein wirksames Werkzeug zur Verfügung, um den Zeitpunkt und Ablauf der Schweinegeburten zu stimulieren.

\section{Literatur}

BERNHARD, A.; SCHULZ, J.; GUTJAHR, S.; EULENBERGER, K.:

Indikationen für die Anwendung eines Depotoxytozin-Präparates in der tierärztlichen Praxis. Tierärztl. Umschau, Konstanz 48 (1993), 446-453

BILKEI, G.:

Der Einfluss der Biotechnik auf die Reproduktion in Schweinezuchtbeständen. Der praktische Tierarzt, Hannover 74 (1993) 1, 33-38

BUSCH, W.:

Betreuungsmaßnahmen an hochträchtigen, gebärenden und puerperalen Sauen in Großbeständen. In: BUSCH, W.; SCHULZ, J. (Hrsg.): Geburtshilfe bei Haustieren. G. Fischer Verlag Jena, Stuttgart (1993), 500-509

DANNENBERG, H.-D.:

Schweinekrankheiten. 3. überarb. Aufl., Deutscher Landwirtschaftsverlag, Berlin (1986), 258-263

ELLENDORFF, F.:

Neuere Erkenntnisse zur Geburtsphysiologie beim Schwein - Bedeutung für den Geburtsablauf. Der Tierzüchter, Hannover 32 (1980) 4, 138-140

EULENBERGER, K.; SCHULZ, J.; GUTJAHR, S.; STROHBACH, U.; STROHBACH, C.; RANDT, A.: Beeinflussung der Geburt bei Rind und Schwein mit Oxytocin, Carbetocin und Carazolol. Wiener Tierärztl. Monatsschr. 80 (1993), 791-806

HOY, St.:

Untersuchungen zum Einfluss verschiedener Haltungsformen auf die Häufigkeit von Puerperalerkrankungen bei Sauen. Der praktische Tierarzt, Hannover 83 (2002), 990-996

HÜHN, U.; GEY, G.:

Untersuchungen zum Einfluss der Geburtensynchronisation auf tiergesundheitliche Merkmale von Partussauen und Ferkeln. Arch. Tierz., Dummerstorf 42 (1999), 353-363

KÖNIG, I.; HÜHN, U.:

Zur Steuerung der Fortpflanzung bei Sauen - Eine Retrospektive. Arch. Tierz., Dummerstorf 40 (1997), 239-256

KUDLAČ, E.:

Einsatz von synthetischen hypothalamischen Peptiden und $\mathrm{PGF}_{2 \alpha}$-Analoga zur Beeinflussung der Sexualfunktionen bei weiblichen Haustieren. Tierärztl. Umschau, Konstanz 48 (1993), 26-29

LEHMANN, H. D.:

Pharmakologische Aspekte der Geburtshilfe bei Zuchtsauen. Tierärztl. Umschau, Konstanz 39 (1984), 487-493

MAFFEO, G.; GEROLDI, S.; CERATI, C.; SALVO, R.; NISOLI, L. G. C.; COLOMBANI, C.; VIGO, D.: 
The use of carbetocine in sows at the beginning of farrowing. Proc. II. $12^{\text {th }}$ IPVS Congress, The Hague (1990), 17-20

MOTA-ROJAS, D.; MARTINEZ-BURNES, J.; TRUJILLO-ORTEGA, M. E.; ALONSO-SPILSBURY, L.; RAMIREZ-NECOECHEA, R.; LOPEZ, A.:

Effect of oxytocin treatment in sows on umbilical morphology, meconium staining, and neonatal mortality of piglets. Am. J. Vet. Res. 63 (2002), 1571-1574

PLONAIT, K.:

Geburt, Puerperium und perinatale Verluste. In: WALDMANN, K.H.; WENDT, M. (Hrsg.): Lehrbuch der Schweinekrankheiten. 3. durchgesehene Aufl., Parey Buchverlag Berlin (2001), 471-507

PRANGE, H.:

Geburten gewissenhaft vorbereiten und engagiert überwachen. Schweinezucht und Schweinemast, Münster 49 (2001) 1, 36-39

PRANGE, H.:

Zur Tiergesundheit von Sau und Ferkeln im geburtsnahen Zeitraum. REKASAN ${ }^{\circledR}$-Journal, Kaulsdorf/Thür., 9 (2002), 104-108

REICHEL, F.:

Depotocin inj. Spofa (carbetocin) v biotechnice reprodukce. Biol. Chem. Ziv. Vet., Praha 24 (1988), 497-503

REIMANN, Th.:

Ergebnisse "Forum Spitzenbetriebe Ferkelproduktion, Schweinemast". Tagungsunterlagen 1. Blockfortbildung "Integrierte Tierärztliche Bestandsbetreuung beim Schwein", 27. bis 30. März 2003, Göttingen

SCHULZ, G.:

Kurze Geburt - vitalere Ferkel. dlz-agrarmagazin, München 54 (2002) 11, 106-110

SPITSCHAK, K.; HÜHN, U.:

Untersuchungen zum Geburtsgeschehen bei Altsauen nach Depotocinbehandlung. Arch. Tierz., Dummerstorf 37 (1994) Sonderheft, 165

THORUP, F.:

Kann die Anzahl totgeborener Ferkel je Wurf reduziert werden? Wiss. Beiträge 8. Bernburger Biotechnik-Workshop, Tag.-Band Hochschule Anhalt (2002), 45-48

UDLUFT, K.:

Erfahrungen bei der Durchführung der Geburtensynchronisation unter großbetrieblichen Produktionsbedingungen. Wiss. Beiträge zur Fachtagung Geburtensynchronisation beim Schwein am 28./29. April 1988 in Burkersdorf, Tag.-Band FZ für Tierproduktion Dummerstorf (1988), 50-63

UDLUFT, T.; BOSTEDT, H.:

Einfluss geburtssteuernder Maßnahmen auf die Ferkelverluste. Vortrag, 9. Bernburger BiotechnikWorkshop, Tag.-Band Hochschule Anhalt (2003), 93

UDLUFT, T.; BOSTEDT, H.: Influence of control measures on parturition in sows. Vet. Med. Austria, Wien 91 (2004), Suppl. 2, 68

WÄHNER, M.; HÜHN, U.: Control of parturition in sows by using a combined treatment with Cloprostenol plus Depotocin ${ }^{\circledR}$. Arch. Tierz., Dummerstorf 44 (2001) Special Issue, 151-154

WENDT, M.:

Management im Abferkelstall zur Vermeidung von MMA-Erkrankungen. Zuchtwahl und Besamung, Neustadt a.d. Aisch (2002), 147: 55-57

ZAREMBA, W.; HÜHN, U.:

Zum Einfluss der biotechnischen Geburtensteuerung auf Merkmale der Wurfqualität bei Altsauen. Züchtungskunde, Stuttgart 71 (1999), 371-379

Eingegangen: 08.06.2004

Akzeptiert: 23.09.2004

Anschriften der Verfasser

Prof. Dr. UWE HÜHN

An der Romenei 7

D-98617 Wölfershausen

PD Dr. habil. AXEL WEHREND

Frankfurter Straße 106

D-35392 Gießen

Tierarzt THOMAS UDLUFT

Thiemsburger Weg 44

PD Dr. Dr. habil. WOLFGANG ZAREMBA

D-99947 Bad Langensalza

Werner-Forßmann-Straße 10

D-34576 Homberg / Efze 